\title{
双核离子液体的合成及其对酯化反应的催化活性
}

\author{
赵地顺*,a 刘猛帅 ${ }^{a}$ 葛京京 ${ }^{a}$ 张 娟 ${ }^{a}$ 任培兵 ${ }^{a, b}$ \\ ( ${ }^{a}$ 河北科技大学化学与制药工程学院 石家庄 050018) \\ ( ${ }^{b}$ 石家庄焦化集团有限责任公司 石家庄 050031)
}

\begin{abstract}
摘要 合成了双-(3-甲基-1-咪唑)亚丁基双对甲苯磺酸盐(Im-PTSA)、双-(3-甲基-1-咪唑)亚丁基双硫酸氢盐(Im-HSO 4 )、 双-(1-吡啶)亚丁基双对甲苯磺酸盐(Py-PTSA)、双-(1-吡啶)亚丁基双硫酸氢盐 $\left(\mathrm{Py}-\mathrm{HSO}_{4}\right.$ )等 4 种功能化双核离子液体. 分 别采用红外光谱(FT-IR)、核磁共振氢谱 ${ }^{1} \mathrm{H}$ NMR) 对合成的离子液体进行结构分析; 采用热重(TG)测试了离子液体的热 稳定性; 此外, 考察了离子液体的酸性和溶解性. 以丁二酸和乙醇的醌化反应考察了 4 种离子液体的催化活性, 结果表 明: 当 $n\left(\mathrm{C}_{4} \mathrm{H}_{6} \mathrm{O}_{4}\right): n\left(\mathrm{C}_{2} \mathrm{H}_{5} \mathrm{OH}\right)=1: 3$, 催化剂 Im-PTSA 量占总质量的 $1.90 \%$, 反应温度 $70{ }^{\circ} \mathrm{C}$, 反应时间 $2.5 \mathrm{~h}$, 酯收率 可达 $93.6 \%$, 选择性达 $100 \%$, 且离子液体经真空干燥重复使用 8 次, 催化活性没有明显降低. 以奥氏体 $316 \mathrm{~L}$ 不锈钢为 试样, 考察了双核功能化离子液体的腐蚀性, 与浓硫酸进行对比, 其对钢试样的腐蚀率不到浓硫酸的 1/10. 以双-(3-甲 基-1-咪唑)亚丁基双对甲苯磺酸盐(Im-PTSA)为催化剂, 考察了一元有机酸和二元有机酸与系列醇的酯化反应, 均获得 较高的酯收率和选择性, 反应结束后产品与催化剂自动分层, 简化了分离, 有望成为一种具有发展潜力的酯化催化剂. 关键词 双核离子液体; 醌化反应; 催化剂; 丁二酸二乙酯; 腐蚀性
\end{abstract}

\section{Synthesis of Binuclear Ionic Liquids and Their Catalytic Activity for Esterification}

\author{
Zhao, Dishun $^{*, a} \quad$ Liu, Mengshuai $^{a} \quad$ Ge, Jingjing ${ }^{a} \quad$ Zhang, Juan $^{a} \quad$ Ren, Peibing ${ }^{a, b}$ \\ ( ${ }^{a}$ College of Chemical and Pharmaceutical Engineering, Hebei University of Science and Technology, Shijiazhuang 050018) \\ ( ${ }^{b}$ Shijiazhuang Coking and Chemical Group Co., Ltd., Shijiazhuang 050031)
}

\begin{abstract}
A series of functional binuclear ionic liquids based on bis-(3-methyl-1-imidazole)butylidene double P-toluene sulfonic acid salt (Im-PTSA), bis-(3-methyl-1-imidazole)butylidene double bisulfate ( $\left.\mathrm{Im}-\mathrm{HSO}_{4}\right)$, bis-(1-pyridine)butylidene double $p$-toluene sulfonic acid salt (Py-PTSA), bis-(1-pyridine)butylidene double bisulfate $\left(\mathrm{Py}-\mathrm{HSO}_{4}\right)$ were synthesized by a two-step proceeding and their structures were characterized by FT-IR and ${ }^{1} \mathrm{H}$ NMR spectra. Their thermal stabilities were characterized by TG. In addition, the acidity and solubility of functional binuclear ionic liquids were also studied. The catalytic activity of the binuclear ionic liquids for the esterification of succinic acid with ethanol was measured. The results show that under the optimized conditions of $n$ (succinic acid) $: n$ (ethanol) $=1: 3$, catalyst used dosage $1.90 \%(\mathrm{wt}), 70{ }^{\circ} \mathrm{C}$ and $2.5 \mathrm{~h}$, the yield of diethyl succinate reached $93.6 \%$ and the selectivity was near up to $100 \%$. Im-PTSA was reused at least 8 times without significant decrease in activity after drying under vacuum. Austenitic stainless steel $316 \mathrm{~L}$ was used for conducting the corrosion test under the above esterificaion condition, the corrosion rates of the steel plates dipped in the systems with these ionic liquids were less than one tenth of that with sulfuric acid. Fischer esterification of monocarboxylic acids and dicarboxylic acids with different alcohols was observed on using Im-PTSA as catalyst which gave high product yield and selectivity. Use of such a reaction catalyst should be appreciated for its convenient separation.
\end{abstract}

Keywords binuclear ionic liquid; esterification; catalyst; diethyl succinate; corrosion

有机羧酸酯是一类应用广泛的重要精细化工产品, 主要用于溶剂、增塑剂、树脂、涂料、香料、化妆品、
医药、表面活性剂及有机合成工业 ${ }^{[1 \sim 4]}$. 传统合成方法 主要是浓硫酸催化法, 该工艺比较成熟, 为人类源源不

\footnotetext{
*E-mail: zhao_dsh@hebust.edu.cn; Tel.: 0311-88632231; Fax: 0311-88632231.

Received July 2, 2012; revised August 3, 2012; published online August 10, 2012.

Project supported by the National Natural Science Foundation of China (Nos. 20576026, 21106032) and the Scientific and Technological Innovation of Hebei University of Science and Technology (2012).

国家自然科学基金(Nos. 20576026, 21106032)、河北科技大学 2012 年度大学生科技创新基金资助项目.
} 
断提供有机羒酸酯的同时, 造成了环境破坏, 威胁着人 类健康. 随着对催化剂的广泛研究, 出现了固体超强 酸、杂多酸、对甲苯磺酸以及强酸性阳离子交换树脂等 多种酯化催化剂, 但普遍存在催化活性低、反应温度高、 易失活、回收困难等问题, 限制了其应用. 离子液体作 为一种新型的绿色催化剂, 在很多酸催化反应中表现出 优异的特性 ${ }^{[5-9]}$. Deng 等 ${ }^{[10]}$ 首先报道了使用吡啶氯铝酸 类离子液体作为催化剂应用于酯化反应, 但由于其对水 和空气不稳定, 而酯化过程又有水产生, 限制了其应用. Fraga-Dubreuil 等 ${ }^{[11]}$ 研究了耐水系离子液体 [Bmim]$\left[\mathrm{HSO}_{4}\right], \quad[\mathrm{Hmim}]\left[\mathrm{HSO}_{4}\right], \quad[\mathrm{Bmim}]\left[\mathrm{H}_{2} \mathrm{PO}_{4}\right], \quad[\mathrm{Heemim}]-$ $\left[\mathrm{HSO}_{4}\right]$ 在酯化反应中的催化活性, 表现出较好的催化性 能, 突出的问题是酸性弱, 低催化用量下催化活性有限, 有待进一步开发. 2002 年, Cole 等 ${ }^{[12]}$ 首先制备出了具有 强 Brønsted 酸性的磺酸基功能化的咪唑盐和季鏻盐离 子液体. 从研究结果来看, 酯化催化剂大部分为烷基磺 酸功能化离子液体, 其 Brønsted 酸性与硫酸几乎相当, 设备腐蚀问题同样较为严重, 成为工业实验过程中生产 装置选择的一大障碍. 寇元等 ${ }^{[13]}$ 提出针对特定的应用 目的量身定制离子液体, 开发具有工业应用前景的功能 化酸性离子液体的研究思路.

目前，对于双核功能化离子液体(IL)的开发还相对 较少. 双核离子液体是一类稳定性更高、具有温控特性 的离子液体, 将特定的双阳离子核与无机/有机酸阴离 子作用, 可以调节无机/有机酸的催化性能. 双核离子液 体应用于酯化反应中, 反应条件温和, 产品收率高, 对 设备腐蚀性较小, 且催化剂易于回收. 我们制备了 4 种 新型双核阳离子离子液体双-(3-甲基-1-咪唑)亚丁基双 对甲苯磺酸盐(Im-PTSA)、双-(3-甲基-1-咪唑)亚丁基双 硫酸氢盐 $\left(\mathrm{Im}-\mathrm{HSO}_{4}\right)$ 、双-(1-吡啶)亚丁基双对甲苯磺酸盐 (Py-PTSA)、双-(1-吡啶)亚丁基双硫酸氢盐 $\left(\mathrm{Py}-\mathrm{HSO}_{4}\right)$. 对 4 种离子液体的结构和热稳定性进行了表征, 测定了 其酸性和腐蚀性. 考察了离子液体对合成丁二酸二乙酯 反应的催化效果, 并对酯化条件进行了优化. 同时考察 了双-(3-甲基-1-咪唑)亚丁基双对甲苯磺酸盐(Im-PTSA) 对系列一元有机羒酸酯、二元有机羧酸酯的合成, 表现 出较强的催化性能, 克服了传统催化剂存在的缺点, 避 免了挥发性有机溶剂的使用, 提高了生产效率, 降低了 生产成本, 具有广阔的应用前景.

\section{1 结果与讨论}

\section{1 离子液体的结构}

对所合成的 4 种目标化合物(结构如图 1)进行表征. 双核咪唑类离子液体的 FT-IR ( $\mathrm{KBr}$ 压片)谱图见图 2. $3101 \mathrm{~cm}^{-1}$ 左右为芳环的 $=\mathrm{C}-\mathrm{H}$ 伸缩振动峰, 2970 ,
$2882 \mathrm{~cm}^{-1}$ 附近出现了 $\mathrm{CH}_{3}$ 上 $\mathrm{C}-\mathrm{H}$ 的非对称和对称伸 缩振动峰, 1573.71 和 $1396.03 \mathrm{~cm}^{-1}$ 为咪唑环 $\mathrm{C}=\mathrm{N}$ 伸缩 振动, $1487 \mathrm{~cm}^{-1}$ 附近出现的吸收峰对应于 $\mathrm{CH}_{2}$ 的弯曲振 动, $756.06 \mathrm{~cm}^{-1}$ 为咪唑环弯曲振动峰, $\mathrm{HSO}_{4}^{-}$和 $\mathrm{SO}_{3} \mathrm{H}$ 在 $1900 \mathrm{~cm}^{-1}$ 附近和 $1150 \sim 1050 \mathrm{~cm}^{-1}$ 处有两个强吸收 峰, $650 \sim 575 \mathrm{~cm}^{-1}$ 处有中等吸收峰. ${ }^{1} \mathrm{H}$ NMR $\left(\mathrm{D}_{2} \mathrm{O}, 500\right.$ MHz) $\delta: 1.78\left(\mathrm{~m}, 4 \mathrm{H}, \mathrm{CH}_{2}\right), 3.75\left(\mathrm{~s}, 6 \mathrm{H}, \mathrm{CH}_{3}\right), 4.11(\mathrm{t}, J=$ $\left.4.6 \mathrm{~Hz}, 4 \mathrm{H}, \mathrm{CH}_{2}\right), 7.32(\mathrm{~d}, J=2.0 \mathrm{~Hz}, 2 \mathrm{H}), 7.36$ (d, $J=1.9$ $\mathrm{Hz}, 2 \mathrm{H}), 8.60$ (s, 2H). IR 和 ${ }^{1} \mathrm{H}$ NMR 表征结果确定所分 析离子液体为目标产物.
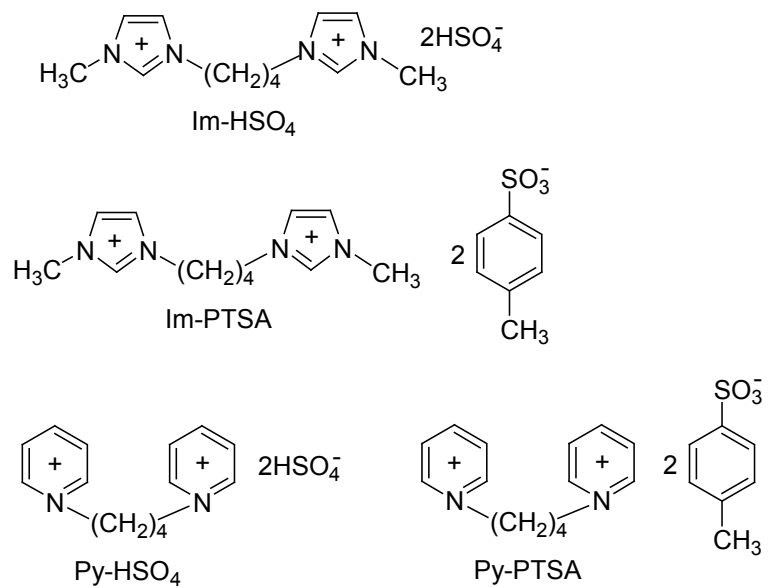

图 1 离子液体的结构示意图

Figure 1 Structure of binuclear ionic liquids

双核吡啶类离子液体的 FT-IR ( $\mathrm{KBr}$ 压片)谱图见图 2. 在 $3136.16 \mathrm{~cm}^{-1}$ 处有芳环上 $\mathrm{C}-\mathrm{H}$ 伸缩振动吸收峰, $3069.15,1585.03,1635.95 \mathrm{~cm}^{-1}$ 附近分别为芳香环上的 $\mathrm{C}=\mathrm{C}-\mathrm{H}, \mathrm{C}=\mathrm{N}, \mathrm{C}=\mathrm{C}$ 伸缩振动峰, $1489.89 \mathrm{~cm}^{-1}$ 处有 $\mathrm{CH}_{2}$ 伸缩吸收峰, $780.11 \mathrm{~cm}^{-1}$ 处有吡啶环上 $\mathrm{C}=\mathrm{C}$ 弯曲 振动峰, $1122 \mathrm{~cm}^{-1}$ 处强吸收峰是磺酸基不对称伸缩峰, 1034, $1011 \mathrm{~cm}^{-1}$ 处的强吸收峰是磺酸基的对称伸缩峰; ${ }^{1} \mathrm{H}$ NMR $\left(\mathrm{D}_{2} \mathrm{O}, 500 \mathrm{MHz}\right) \delta: 2.06(\mathrm{~s}, 4 \mathrm{H}), 4.65(\mathrm{~s}, 4 \mathrm{H})$, $8.01(\mathrm{t}, J=4.9 \mathrm{~Hz}, 4 \mathrm{H}), 8.51(\mathrm{t}, J=3.8 \mathrm{~Hz}, 2 \mathrm{H}), 8.78(\mathrm{~d}$, $J=2.6 \mathrm{~Hz}, 4 \mathrm{H})$; IR 和 ${ }^{1} \mathrm{H} \mathrm{NMR}$ 表征结果确定所分析离子 液体为目标产物.

\section{2 离子液体的热稳定性}

由图 3 可知, 4 种双核功能化离子液体在 $250{ }^{\circ} \mathrm{C}$ 以 下几乎没有热失重, 于一般的酸醇酯化反应温度 $(\leqslant 150$ ${ }^{\circ} \mathrm{C}$ ) 条件下均具有较高的热稳定性. 图中 4 种离子液体 开始失重温度差别不大, 均为 $275{ }^{\circ} \mathrm{C}$ 左右; 而离子液体 最大失重速率温度值 $\left(\mathrm{DTG}_{\max }\right)$ 差别较大, $\mathrm{Py}-\mathrm{HSO}_{4}$,

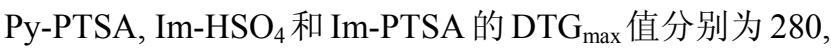
303，325 和 $324{ }^{\circ} \mathrm{C}$. 阴离子相同的双核吡啶离子液体 $\mathrm{DTG}_{\max }$ 比 $N$-甲基咪唑离子液体低, 可能是由于吡啶杂 


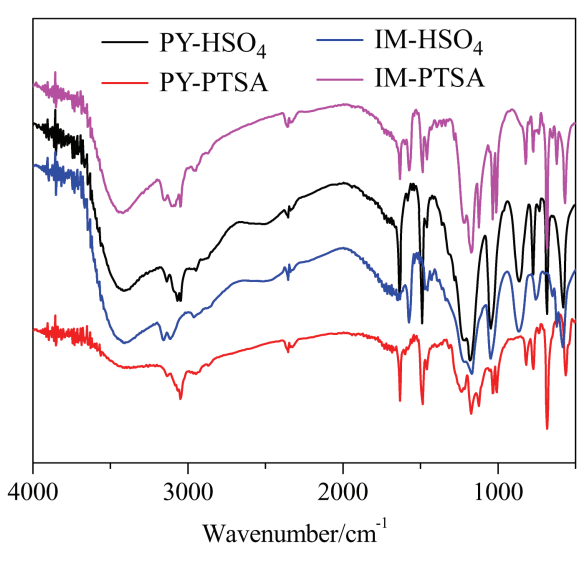

图 2 离子液体的红外谱图

Figure 2 IR spectra of ionic liquids

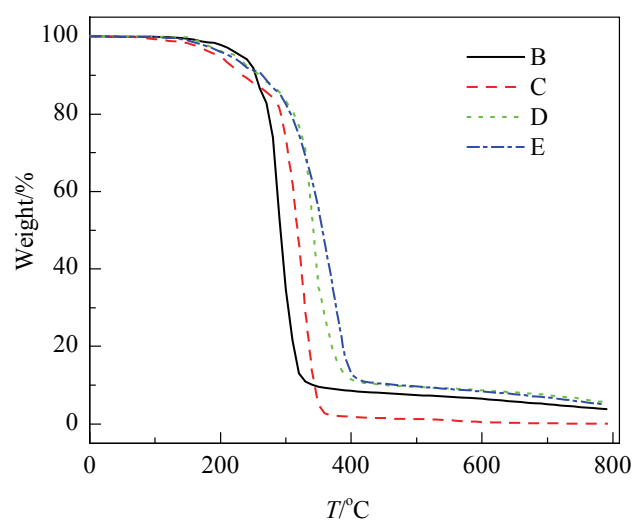

图 3 离子液体的热稳定性

Figure 3 TG curves of ionic liquids

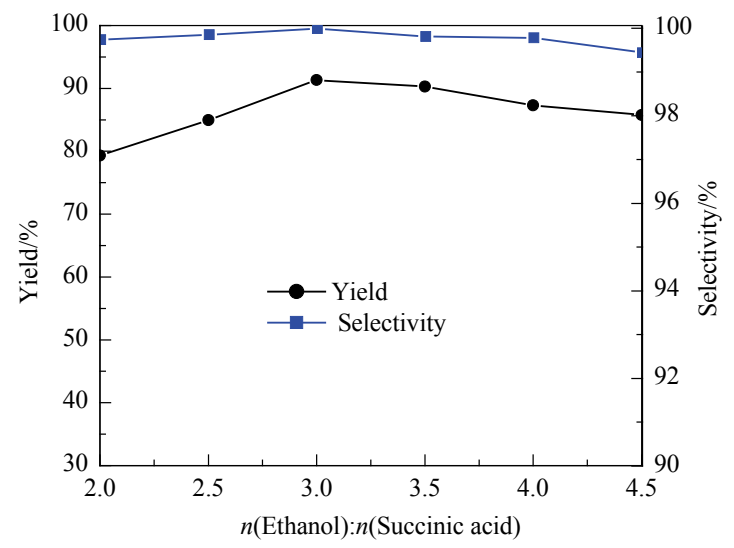

图 4 醇酸比对酯化反应的影响

Figure 4 Effects of molar ratio of acid to alcohol on the yield of esterification

Reaction conditions: $n$ (succinic acid) $=0.05 \mathrm{~mol}$, mass fraction of catalyst $1.88 \%, T=80{ }^{\circ} \mathrm{C}, t=2 \mathrm{~h}$

环较咪唑环与阴离子之间的距离大, 从而导致引力减 小, 在受热过程中较容易分解; 相同阳离子不同阴离子 的两类离子液体的热稳定性却呈现不同的规律，表明离 子液体的热稳定性分别受到杂原子-碳原子之间作用力 和杂原子-氢键之间作用力的限制，与组成离子液体的
阳离子和阴离子的结构和性质密切相关 ${ }^{[14]}$.

\section{3 离子液体的酸性}

表 1 为不同浓度(质量分数)的离子液体与浓硫酸的 水溶液的 $\mathrm{pH}$ 值. 可以看出, 相同浓度下，离子液体的酸 性顺序为: $\mathrm{H}_{2} \mathrm{SO}_{4}>\mathrm{Im}-\mathrm{HSO}_{4}>\mathrm{Py}-\mathrm{HSO}_{4}>\mathrm{Im}-\mathrm{PTSA}>$ Py-PTSA. 阴离子为 ${ }^{-} \mathrm{HSO}_{4}$ 的双核功能化离子液体酸性 明显强于阴离子为 ${ }^{-} \mathrm{PTSA}$ 的离子液体, 质量浓度为 $1 \%$ 的水溶液, $\mathrm{Im}-\mathrm{HSO}_{4}, \mathrm{Py}-\mathrm{HSO}_{4}$ 的 $\mathrm{pH}$ 值分别为 1.49 和 1.54 , 表现出较强的 Brønsted 酸性. 当离子液体的阴离 子相同时，双核咪唑类离子液体酸性略强于双核吡啶类 离子液体, 其阳离子结构对离子液体的 Brønsted 酸性影 响较小, 阳离子的酸性越强, 其 Brønsted 酸性越强; 当 阳离子相同时，阴离子活性顺序为 ${ }^{-} \mathrm{HSO}_{4}>^{-} \mathrm{PTSA}$, 这 与阴离子同质子结合的能力有关, 容易与质子结合, 表 明其碱性较强, 而其共轭酸的酸性较弱, 说明阴离子对 双核功能化离子液体的酸性影响较大.

表 1 离子液体的酸性 ${ }^{a}$

Table 1 Acidity of various ionic liquids in water

\begin{tabular}{cccccc}
\hline \multirow{2}{*}{ Ionic liquid } & \multicolumn{5}{c}{$\mathrm{pH}$} \\
\cline { 2 - 6 } & $1 \%$ & $2 \%$ & $3 \%$ & $4 \%$ & $5 \%$ \\
\hline Py-HSO & 1.54 & 1.31 & 1.12 & 0.98 & 0.91 \\
Py-PTSA & 1.85 & 1.61 & 1.38 & 1.18 & 1.03 \\
Im-HSO $_{4}$ & 1.49 & 1.23 & 1.04 & 0.90 & 0.81 \\
Im-PTSA & 1.60 & 1.32 & 1.13 & 1.00 & 0.94 \\
$\mathrm{H}_{2} \mathrm{SO}_{4}$ & 0.96 & 0.75 & 0.58 & 0.44 & 0.35 \\
\hline
\end{tabular}

${ }^{a}$ Test condition: $T=20{ }^{\circ} \mathrm{C}$.

\section{4 离子液体的溶解性}

离子液体的溶解性能是其在使用过程中的一项重 要性质, 选取 $2 \mathrm{~mL}$ 离子液体, 在室温下加入溶剂中, 震 荡、搅拌, 考察了不同双核功能化离子液体分别在常见 有机溶剂中的溶解性, 结果见表 2 .

离子液体完全是由离子组成的, 按照相似相溶理 论，离子液体应该溶解强极性或离子组分. 从表 2 看出, 各离子液体均溶于强极性的水, $\mathrm{Py}-\mathrm{HSO}_{4}, \mathrm{Py}-\mathrm{PTSA}$, Im-PTSA 与极性较强的乙腈互溶, 与其他极性溶剂均出 现了不互溶的现象, 和常见离子液体略有不同. 离子液 体特殊的溶解性决定了该类离子液体适合作为催化剂 应用于有机合成反应中，可以简化分离过程. 尤其应用 于有水生成的酯化反应中, 不但催化剂回收方便, 而且 有利于反应的进行.

\section{5 离子液体的腐蚀性}

腐蚀性作为离子液体的一个重要性质, 在很大程度 上决定了离子液体催化酯化反应过程的安全性和经济 性 ${ }^{[15]}$, 表 3 给出了 $316 \mathrm{~L}$ 不锈钢板在离子液体和浓硫酸 催化的酯化体系中的腐蚀率. 当催化剂用量均占反应体 
表 2 离子液体的溶解性 ${ }^{a}$

Table 2 Solubility of various acidic ionic liquids

\begin{tabular}{|c|c|c|c|c|c|c|}
\hline \multirow{2}{*}{ Ionic liquid } & \multicolumn{6}{|c|}{ Solubility of solvent } \\
\hline & Water & Acetone & Ether & Ethanol & Dichloromethane & Cyclohexane \\
\hline $\mathrm{Py}-\mathrm{HSO}_{4}$ & Soluble & Insoluble & Insoluble & Insoluble & Insoluble & Insoluble \\
\hline Py-PTSA & Soluble & Soluble & Insoluble & Soluble & Insoluble & Insoluble \\
\hline $\mathrm{Im}-\mathrm{HSO}_{4}$ & Soluble & Insoluble & Insoluble & Insoluble & Insoluble & Insoluble \\
\hline Im-PTSA & Soluble & Insoluble & Insoluble & Soluble & Insoluble & Insoluble \\
\hline $\mathrm{H}_{2} \mathrm{SO}_{4}$ & Soluble & Reaction & Reaction & Reaction & Soluble & Soluble \\
\hline \multirow{2}{*}{ Ionic liquid } & \multicolumn{6}{|c|}{ Solubility of solvent } \\
\hline & Benzol & Methylbenzene & Acetonitrile & $n$-Hexane & Trichloromethane & Acetic ether \\
\hline $\mathrm{Py}-\mathrm{HSO}_{4}$ & Insoluble & Insoluble & Soluble & Insoluble & Insoluble & Insoluble \\
\hline Py-PTSA & Insoluble & Insoluble & Soluble & Insoluble & Insoluble & Insoluble \\
\hline $\mathrm{Im}-\mathrm{HSO}_{4}$ & Insoluble & Insoluble & Insoluble & Insoluble & Insoluble & Insoluble \\
\hline Im-PTSA & Insoluble & Insoluble & Soluble & Insoluble & Insoluble & Insoluble \\
\hline $\mathrm{H}_{2} \mathrm{SO}_{4}$ & Reaction & Soluble & Reaction & Insoluble & Insoluble & Soluble \\
\hline
\end{tabular}

${ }^{a}$ Test conditions: $V($ solvent $)=5 \mathrm{~mL}, m(\mathrm{IL})=0.25 \mathrm{~g}, m\left(\mathrm{H}_{2} \mathrm{SO}_{4}\right)=0.25 \mathrm{~g}$, r.t.

表 3 离子液体的腐蚀性

Table 3 Corrosion of ILs for Austenitic stainless steel 316 after immersion

\begin{tabular}{clccccc}
\hline Entry & Ionic liquid & $m$ (Ionic liquid) $/ \mathrm{g}$ & $t / \mathrm{h}$ & $T /{ }^{\circ} \mathrm{C}$ & Mass loss $/ \mathrm{g}$ & ${\text { Corrosion rate } /\left(\mathrm{g}^{\circ} \mathrm{m}^{-2} \cdot \mathrm{h}^{-1}\right)}^{2.15}$ \\
\hline 1 & Py-HSO & 12 & 1.00 & 12 & 0.0217 & 1.72 \\
2 & Py-PTSA & 1.00 & 12 & 80 & 0.0173 & 2.38 \\
3 & $\mathrm{Im}-\mathrm{HSO}_{4}$ & 1.00 & 12 & 80 & 0.0240 & 1.69 \\
4 & $\mathrm{Im}-\mathrm{PTSA}_{5}$ & 1.00 & 12 & 80 & 0.0170 & 17.60 \\
\hline
\end{tabular}

Test conditions: $n($ ethanol $)=0.6 \mathrm{~mol}, n($ succinic acid $)=0.2 \mathrm{~mol}$, mass fraction of catalyst $1.92 \%$

系总质量 $1.92 \%$ 时, 浸渍在 $\mathrm{Py}-\mathrm{HSO}_{4}, \mathrm{Im}-\mathrm{HSO}_{4}, \mathrm{H}_{2} \mathrm{SO}_{4}$ 催化体系中的 $316 \mathrm{~L}$ 不锈钢板试样表面都有气泡冒出, 但 $\mathrm{H}_{2} \mathrm{SO}_{4}$ 催化体系中的反应现象明显比离子液体催化 体系剧烈, 浸渍 $12 \mathrm{~h}$ 后试样表面颜色加深, 有许多蚀点; 在离子液体 Py-PTSA, Im-PTSA 催化体系中没有观察到 明显的反应现象, 浸渍后试样表面没有明显的变化, 质 量只有微量损失. 从表 3 中可以看出, 阴离子为 ${ }^{-} \mathrm{HSO}_{4}$ 的离子液体在催化反应过程中对设备的腐蚀性明显强 于阴离子为 ${ }^{-}$PTSA 的离子液体, 原因除了酸性较强的 原因外还可能与阴离子在催化体系中发生水解有关 ${ }^{[16]}$; 阴离子同为 ${ }^{-}$PTSA 的双核咪唑离子液体较吡啶离子液 体腐蚀性更低, 腐蚀率不到浓硫酸的 $1 / 10$, 说明双核功 能化咪唑类离子液体比双核吡啶类离子液体对水的稳 定性更强.

\section{6 离子液体的催化活性}

在相同反应条件下, 分别考察 4 种双核功能化离子 液体作为催化剂对丁二酸和乙醇反应合成丁二酸二乙 酯的影响, 结果见表 4 中 Entries 1 4. 作为对比, 同时 考察了单核离子液体和浓硫酸作为催化剂, 在醇酸物质 的量比为 $3: 1$, 催化剂量为反应体系总质量的 $2 \%$ (质量 分数), 反应时间 $3 \mathrm{~h}$, 反应温度 $80^{\circ} \mathrm{C}$ 条件下对产品收率 的影响, 见表 4 中 Entries $5 \sim 9$.
表 4 离子液体的催化活性

Table 4 Effect of different catalysts on results of esterification

\begin{tabular}{|c|c|c|c|c|c|c|c|}
\hline Entry & Ionic liquid & $\begin{array}{c}\text { Molar } \\
\text { ratio }\end{array}$ & $\omega(\mathrm{IL}) / \%$ & $t / \mathrm{h}$ & $T /{ }^{\circ} \mathrm{C}$ & \multicolumn{2}{|c|}{ Yield $/ \%$ Select. $/ \%$} \\
\hline 1 & $\mathrm{Py}-\mathrm{HSO}_{4}$ & $3: 1$ & 2.0 & 3 & 80 & 80.2 & 98.4 \\
\hline 2 & Py-PTSA & $3: 1$ & 2.0 & 3 & 80 & 70.4 & 97.6 \\
\hline 3 & $\mathrm{Im}_{-} \mathrm{HSO}_{4}$ & $3: 1$ & 2.0 & 3 & 80 & 83.2 & 99.3 \\
\hline 4 & Im-PTSA & $3: 1$ & 2.0 & 3 & 80 & 79.9 & 99.9 \\
\hline 5 & {$[\mathrm{BPy}] \mathrm{HSO}_{4}$} & $3: 1$ & 2.0 & 3 & 80 & 72.1 & 98.4 \\
\hline 6 & [BPy]PTSA & $3: 1$ & 2.0 & 3 & 80 & 60.7 & 97.1 \\
\hline 7 & {$[\mathrm{Bmim}] \mathrm{HSO}_{4}$} & $3: 1$ & 2.0 & 3 & 80 & 73.6 & 98.9 \\
\hline 8 & [Bmim]PTSA & $3: 1$ & 2.0 & 3 & 80 & 66.0 & 97.8 \\
\hline 9 & $\mathrm{H}_{2} \mathrm{SO}_{4}$ & $3: 1$ & 2.0 & 3 & 80 & 80.1 & 95.8 \\
\hline
\end{tabular}

由表 4 可见: 双核功能化离子液体和单核离子液体 对丁二酸和乙醇都具有明显的催化作用, 酯的选择性比 浓 $\mathrm{H}_{2} \mathrm{SO}_{4}$ 高. 双核功能化离子液体催化产品收率顺序 为: $\mathrm{Im}-\mathrm{HSO}_{4}>\mathrm{Py}-\mathrm{HSO}_{4}>\mathrm{Im}-\mathrm{PTSA}>\mathrm{Py}-\mathrm{PTSA}$, 这与它 们的酸强度由强到弱的顺序一致, 而离子液体的酸性强 弱受阴离子影响较大, 因此离子液体高酸性的阴离子导 致了高的酯收率 ${ }^{[17]}$. 此外, 反应结束后离子液体均能与 产品酯自动分层, 不仅简化了反应装置, 而且有利于酯 化反应向正方向进行, 这是浓硫酸作催化剂所不能比拟 的. 由于 $\mathrm{Im}-\mathrm{HSO}_{4}, \mathrm{Py}-\mathrm{HSO}_{4}$ 的腐蚀性较 Im-PTSA, Py-PTSA 强很多, 而催化效果相差不大. 因此, 我们选 
择介于酸性和腐蚀性之间的一种温和型离子液体双-(3甲基-1-咪唑)亚丁基对甲苯磺酸盐(Im-PTSA)作为酯化 反应的催化剂进行研究, 以下进一步考察反应条件对酯 化反应的影响.

\section{7 影响酯化反应的因素}

\subsection{1 醇酸物质的量比对酯收率的影响}

图 4 为醇/酸物质的量比对酯收率的影响. 由图可 见, 当醇/酸为理论物质的量比 $2: 1$ 时, 产品收率仅为 $78.8 \%$. 随着 $n$ (乙醇) $: n$ (丁二酸)值的增加, 产品收率和 选择性明显提高; 当 $n$ (乙醇) $: n$ (丁二酸 $)=3$ 时, 酯收率 达到最高, 选择性接近 $100 \%$, 并趋于稳定. 这是因为酯 化反应为可逆反应, 增加原料乙醇的量, 有利于酯化反 应向生成酯的方向移动, 使酯收率和选择性增加. 继续 增大醇/酸比时, 一方面, 反应体系中酸和催化剂的平均 浓度下降，对反应反而不利，导致酯收率和选择性逐渐 下降; 另一方面，乙醇过量太多增加回收成本. 因此， 选择适宜的醇/酸物质的量比为 $3: 1$.

\section{7 .2 催化剂用量对酯收率的影响}

图 5 为催化剂 Im-PTSA 用量对产品收率的影响. 可 以看出, 离子液体 Im-PTSA 对丁二酸二乙酯的合成具 有良好的催化活性, 随着催化剂用量的增加, 酯收率明 显升高, 而后逐渐趋于稳定, 选择性 $\geqslant 96 \%$, 变化较小. 当催化剂用量为 $1.90 \%$ 时, 产品收率及选择性分别达到 93.3\%和 99.8\%，进一步增加催化剂的用量，产品收率和 选择性仍保持在较高水平. 综合考虑产品收率及经济 性, 催化剂用量保持在 $1.90 \%$ 为宜. 表明该离子液体是 一种具有开发潜力的催化剂.

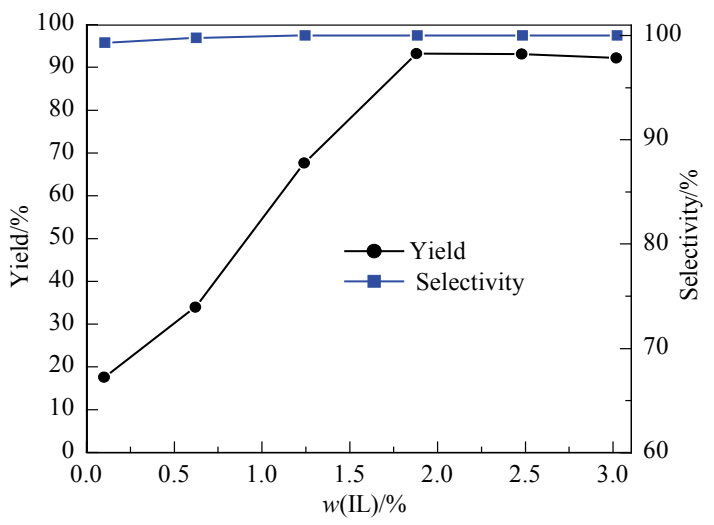

图 5 催化剂用量对酯化反应的影响

Figure 5 Effects of amount of catalyst on the yield of esterification

Reaction conditions: $n$ (succinic acid $)=0.05 \mathrm{~mol}, n$ (ethanol) $: n$ (succinic acid) $=3, T=80{ }^{\circ} \mathrm{C}, t=2 \mathrm{~h}$

\subsection{3 反应温度对酯收率的影响}

图 6 考察了不同反应温度对酯收率的影响. 可以看 出, 随温度升高, 丁二酸二乙酯收率和选择性均呈增大
的趋势，原因是低温条件下，离子液体 Im-PTSA 黏度较 大，反应原料与催化剂不易充分接触; 温度升高时，离 子液体粘度降低, 分子运动加快, 反应进行比较充分. 当反应温度达到 $70{ }^{\circ} \mathrm{C}$ 时, 产品收率达到 $91.4 \%$, 选择 性接近 $100 \%$. 继续升高反应温度，产品收率不再提高， 原因是酯化反应为放热可逆反应，温度过高不利于反应 向正方向移动，故产品收率略有下降. 综合考虑，选择 最佳反应温度为 $70{ }^{\circ} \mathrm{C}$. 与传统方法相比, 功能化离子 液体 Im-PTSA 对丁二酸与乙醇合成丁二酸二乙酯的反 应具有很高的催化活性，在较温和的条件下即可达到酯 化反应的平衡状态.

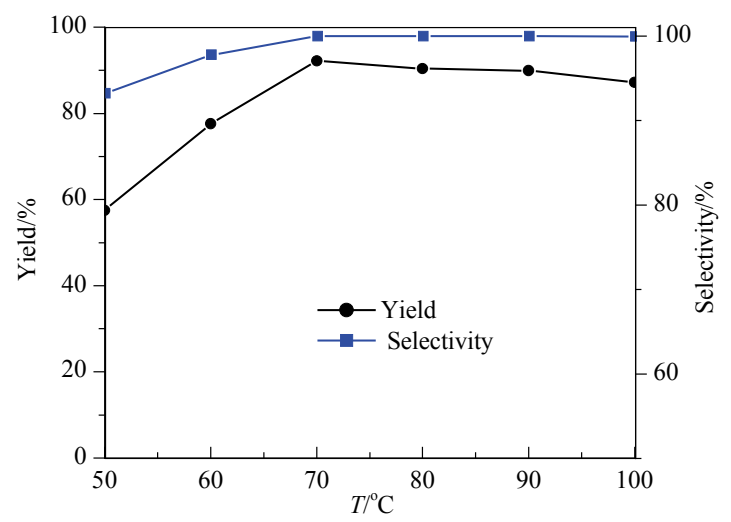

图 6 反应温度对酯化反应的影响

Figure 6 Effects of reaction temperature on the yield of esterification

Reaction conditions: $n$ (succinic acid $)=0.05 \mathrm{~mol}, n$ (ethanol) $: n$ (succinic acid) $=3$, mass fraction of catalyst $1.88 \%, t=2 \mathrm{~h}$

\subsection{4 反应时间对酯收率的影响}

图 7 考察了不同反应时间对酯收率的影响. 可以看 出, 随着反应时间的延长, 酯收率呈先增加后略微下降 的趋势，选择性 $\geqslant 98 \%$. 当反应时间为 $2 \mathrm{~h}$ 时，产品收率 即可达到 $83.1 \%$ ，选择性达 $98.8 \%$. 而当反应时间延长 至 $2.5 \mathrm{~h}$ 时, 酯化反应基本达到平衡, 产品收率增大至 93.6\%, 选择性达到 $100 \%$. 原因是由于离子液体吸收了 反应生成的水，对反应略有推动作用. 继续增大反应时 间，一方面，能耗将增大; 另一方面，产品酯可能会发 生少量水解，导致产品收率下降. 因此，综合考虑选择 最佳反应时间为 $2.5 \mathrm{~h}$. 与传统工艺所需的 $3 \sim 4 \mathrm{~h}$ 相比, 反应时间有所减少.

\subsection{5 催化剂重复使用性能}

在离子液体催化合成丁二酸二乙酯最佳工艺条件 下，即酸醇物质的量比 $1: 3$, 催化剂 Im-PTSA 量占反应 物总质量的 $1.90 \%, 70{ }^{\circ} \mathrm{C}$ 条件下反应 $2.5 \mathrm{~h}$, 反应结束后 离子液体与产品酯自动分层, 经过简单的分液处理即可 实现催化剂的回收. 离子液体经真空干燥除水处理后重 复用于酯化, 结果如图 8. 可以看出, 离子液体在重复 


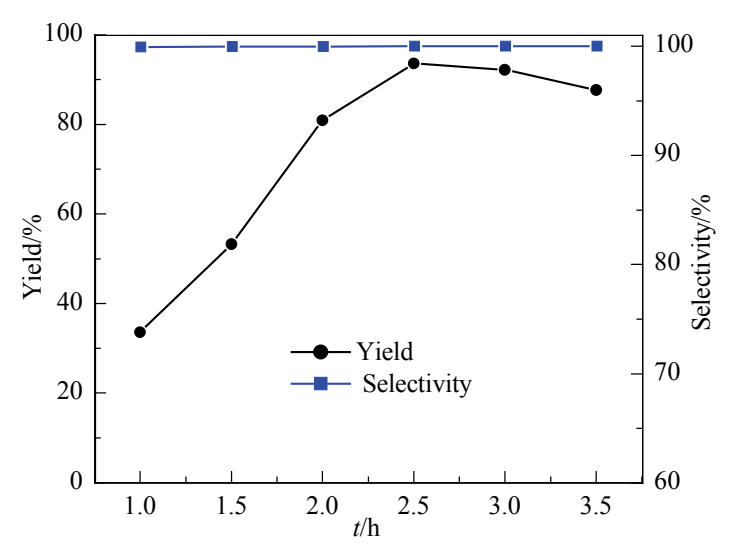

图 7 反应时间对酯化反应的影响

Figure 7 Effects of reaction time on the yield of esterification Reaction conditions: $n$ (succinic acid) $=0.05 \mathrm{~mol}, n$ (ethanol) $: n$ (succinic acid) $=3$, mass fraction of catalyst $1.88 \%, T=70{ }^{\circ} \mathrm{C}$.

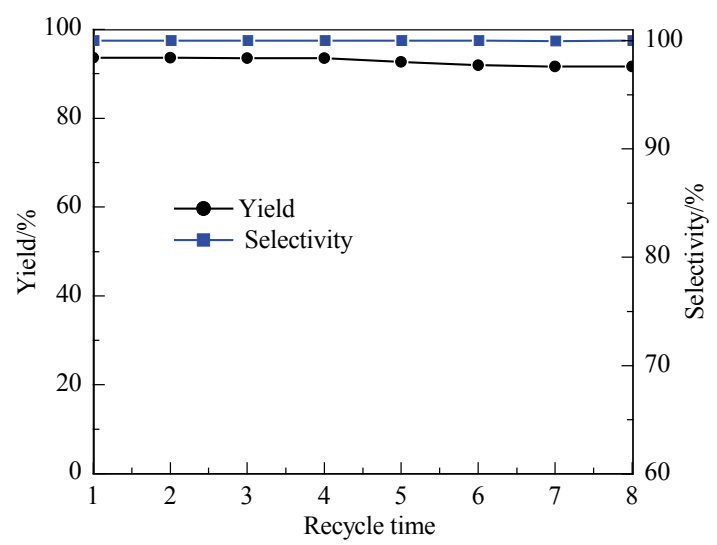

图 8 催化剂的重复使用性能

Figure 8 Recycling ability tests of IL

Reaction conditions: $n$ (succinic acid $)=0.05 \mathrm{~mol}, n$ (ethanol) $: n$ (succinic acid) $=3$, mass fraction of catalyst $1.88 \%, T=70{ }^{\circ} \mathrm{C}, t=2.5 \mathrm{~h}$

使用 8 次后, 活性仅略有下降, 产品的选择性几乎没有 变化. 对重复使用 8 次后的离子液体干燥处理后进行表 征, 发现离子液体的特征峰 $3400(\mathrm{~N}-\mathrm{H}), 3101(=\mathrm{C}-$ $\mathrm{H}), 2970,2882(\mathrm{C}-\mathrm{H}), 1573.71(\mathrm{C}=\mathrm{N}), 1487\left(\mathrm{CH}_{2}\right)$, $1034\left(\mathrm{SO}_{3}\right) \mathrm{cm}^{-1}$ 均没有发生变化, 也没有新的特征峰出 现, 证明反应结束后离子液体的结构和组成并没有发生 变化.

\section{8 离子液体催化合成系列酯}

以双-(3-甲基-1-咪唑)亚丁基对甲苯磺酸盐(Im-
TSA)为催化剂, 研究其对不同酸醇酯化反应的催化效 果, 其中一元有机羧酸酯以醋酸系列酯为例, 二元有机 羧酸酯以丁二酸系列酯为例, 结果如表 5 . 从表中可以 看出, 反应 $2 \sim 4 \mathrm{~h}$, 系类产品酯的收率 $\geqslant 80 \%$, 选择性 接近 $100 \%$, 延长反应时间或升高反应温度可以继续提 高产品收率, 离子液体表现出了较强的催化性能. 反应 结束后, 大部分产品与离子液体完全不互溶, 出现了明 显的分层现象，简化了分离; 另外，在反应过程中生成 的水溶于离子液体，有利于酯化反应向正反应方向进 行, 避免了分水装置的使用, 工艺简单，表现出取代传 统工业催化剂的巨大潜力.

\section{2 结论}

采用两步法成功合成出了双-(3-甲基-1-咪唑)亚丁 基双对甲苯磺酸盐、双-(3-甲基-1-咪唑)亚丁基双硫酸氢 盐、双-(1-吡啶)亚丁基双对甲苯磺酸盐及双-(1-吡啶)亚 丁基双硫酸氢盐 4 种新型功能化离子液体, 在催化酯化 反应中表现出了较强的催化性能. 该新型双核功能化离 子液体与传统酸催化剂相比, 其突出的优点是: (1)酸醇 物质的量比低, 反应温度低, 反应时间短，降低了生产 成本，提高了生产效率; (2)催化剂与产品可以实现自动 分层，避免了分水装置的使用，简化了分离; (3)避免了 挥发性带水剂苯、甲苯、1,2-二氯乙烷等的使用，具有 环境友好性; (4)离子液体腐蚀性比浓 $\mathrm{H}_{2} \mathrm{SO}_{4}$ 低, 副产少, 且容易回收利用，克服了离子液体成本高的问题，减少 了资源的浪费，提高了经济效益. 可见，双核功能化离 子液体是具有工业应用前景的酯化催化剂.

\section{3 实验部分}

\section{1 仪器与试剂}

$N$-甲基咪唑(分析纯，盐城市药物化工厂); 吡啶(分 析纯，天津市大茂化学制剂有限公司)；1,4-二澳丁烷(分 析纯，国药集团上海化学试剂有限公司); 对甲苯磺酸 (分析纯, 天津市永大化学试剂开发中心); 浓硫酸(分析 纯，石家庄炼油厂生产品); 氧化银(分析纯，国药集团 化学试剂有限公司); 乙酸乙酯(分析纯, 天津市永大化

表 5 离子液体催化合成系列酯 ${ }^{a}$

Table 5 Results of esterification of different alcohols and acids with IM-PTSA

\begin{tabular}{cllccccc}
\hline Entry & \multicolumn{1}{c}{ Acid } & \multicolumn{1}{c}{ Alcohol } & Molar ratio & $T /{ }^{\circ} \mathrm{C}$ & $w(\mathrm{IL}) / \%$ & $t / \mathrm{h}$ & Yield/\% \\
\hline 1 & HOAc & Ethanol & $1: 1.2$ & 40 & 3.36 & 2 & 91.7 \\
2 & HOAc & $n$-Propanol & $1: 1.3$ & 60 & 2.82 & 2 & 90.8 \\
3 & HOAc & 1-Butanol & $1: 1.5$ & 70 & 2.29 & 4 & 93.3 \\
4 & Succinic acid & Methyl alcohol & $1: 3.0$ & 80 & 4.46 & 3 & 83.8 \\
5 & Succinic acid & $n$-Propanol & $1: 3.0$ & 80 & 3.25 & 3 & 88.1 \\
6 & Succinic acid & iso-Propyl alcohol & $1: 4.0$ & 90 & 2.72 & 4 & 87.5 \\
\hline
\end{tabular}

${ }^{a}$ Reaction conditions: $\mathrm{HOAc}=$ acetic acid; $n(\mathrm{HOAc})=0.25 \mathrm{~mol} ; n($ succinic acid $)=0.10 \mathrm{~mol}$; ionic liquid $=1 \mathrm{~g}$. 
学制剂有限公司); 丙酮(分析纯, 天津市河东区红岩试 剂厂); 丁二酸(分析纯, 天津市永大化学制剂有限公司); 乙醇(分析纯，天津市永大化学制剂有限公司).

FTS-135 型傅立叶变换红外光谱仪(美国 BIO-RAD 公司); Bruker Avance 型核磁共振仪(瑞士 BRUKER 公 司); TG209 型热重分析仪(德国 Netzsch 公司); GC7890II 型气相色谱仪(上海天美科学仪器有限公司); X-4 型 数字显示显微熔点测定仪 (北京泰克仪器有限公司); PHS-25 型 $\mathrm{pH}$ 计(上海精密科学仪器有限公司).

\section{2 实验方法}

3.2.1 双核离子液体中间体双-(3-甲基-1-咪唑)亚丁 基二溴化盐的制备

称量 $N$-甲基咪唑 $8.21 \mathrm{~g}(0.1 \mathrm{~mol}), 1,4$-二激丁烷 $10.80 \mathrm{~g}(0.05 \mathrm{~mol})$ 于三口烧瓶中混合, 加入溶剂乙腈 80 $\mathrm{mL}$, 冲入 $\mathrm{N}_{2}$ 保护, $80{ }^{\circ} \mathrm{C}$ 条件下冷凝回流 $24 \mathrm{~h}$. 倾倒出 溶剂, 丙酮洗涤 3 次, $80{ }^{\circ} \mathrm{C}$ 下真空 $(0.005 \mathrm{MPa})$ 干燥 $6 \mathrm{~h}$, 得白色固体双-(3-甲基-1-咪唑)亚丁基二溴盐 $17.4 \mathrm{~g}$, 收 率 91.4\%. 反应式见 Eq. 1. 采用同样的方法, 制得双-(1吡啶)亚丁基二溴盐中间体，参照文献[18].

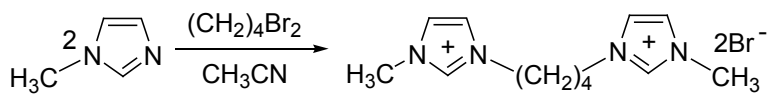

3.2.2 离子液体双-(3-甲基-1-咪唑)亚丁基双对甲苯 磺酸盐的合成

称取该二溴盐 $5.7 \mathrm{~g}(0.015 \mathrm{~mol})$, 对甲苯磺酸 $5.16 \mathrm{~g}$ $(0.030 \mathrm{~mol})$ 溶于 $100 \mathrm{~mL}$ 蒸馏水中, 分批加入 $3.48 \mathrm{~g}$ $\mathrm{Ag}_{2} \mathrm{O}(0.015 \mathrm{~mol})$, 避光反应 $8 \mathrm{~h}$, 过滤除去 $\mathrm{AgBr}$ 沉淀. 乙酸乙酯洗涤 3 次, $80{ }^{\circ} \mathrm{C}$ 真空 $(0.005 \mathrm{MPa})$ 干燥 $12 \mathrm{~h}$, 得 到浅黄色油状液体双-(3-甲基-1-咪唑)亚丁基双对甲苯 磺酸盐 $7.0 \mathrm{~g}$, 产率 $89.8 \%$. 反应式见 Eq. 2 所示, 记作 Im-PTSA. 采用同样的方法合成了双-(3-甲基-1-咪唑)亚 丁基双硫酸氢盐、双-(1-吡啶)亚丁基双对甲苯磺酸盐、 双-(1-吡啶)亚丁基双硫酸氢盐, 分别记作 $\mathrm{Im}-\mathrm{HSO}_{4}$, Py-PTSA, Py-HSO ${ }_{4}$. m.p. 分别为 $-5,-15,40,-12$ ${ }^{\circ} \mathrm{C}$.

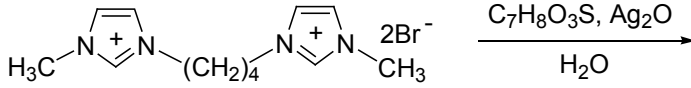

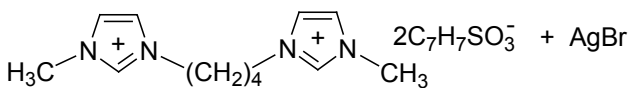

\section{3 酸性测定}

用电子天平分别准确称量上述 4 种双核功能化离子 液体和浓硫酸, 配制各自质量分数分别为 $1 \%, 2 \%, 3 \%$, $4 \%, 5 \%$ 的水溶液, 用 $\mathrm{pH}$ 计测定 $\mathrm{pH}$ 值.

\section{4 酯化反应}

在带有磁力搅拌、回流冷凝管的三口烧瓶中依次加 入丁二酸、无水乙醇、双核功能化离子液体催化剂, 其 中酸醇物质的量比 $1: 3$, 催化剂 $0.3 \mathrm{~g}$ (占总质量的 $2.27 \%), 80{ }^{\circ} \mathrm{C}$ 条件下油浴加热 $2 \mathrm{~h}$, 室温静置; 溶液自 动分层, 上层为酯相, 下层为离子液体相, 分液; 酯层 先后经饱和 $\mathrm{NaCO}_{3}$ 溶液、饱和 $\mathrm{NaCl}$ 溶液洗涤, 加入无 水 $\mathrm{MgSO}_{4}$ 干燥，抽滤、旋蒸得到纯净产物; 离子液体经 简单的减压旋蒸 $\left(80{ }^{\circ} \mathrm{C}\right.$ 、真空度 $\left.0.098 \mathrm{MPa}\right)$ 除水处理后 重复使用.

\section{5 催化剂与产品表征}

利用傅立叶变换红外光谱仪对合成的离子液体及 产品酯进行 IR 测试( $\mathrm{KBr}$ 压片); 在核磁共振仪上进行离 子液体 (溶剂为 $\mathrm{D}_{2} \mathrm{O}$ )及产品酯(溶剂为 $\mathrm{DMSO}$ ) 的 ${ }^{1} \mathrm{H}$ NMR 测定; 利用差热-热重联用热分析仪测试制得离子 液体的热稳定性(热重分析条件为: 升温速率 $10{ }^{\circ} \mathrm{C} /$ $\mathrm{min}$, 炉内动态氮气 $40 \mathrm{~mL} / \mathrm{min}$ ); 利用气相色谱仪测定 产品收率和选择性.

\section{6 腐蚀性考察}

实验采用奥氏体型 $316 \mathrm{~L}$ 不锈钢板作为试验钢样 (上海宝钢集团, $20 \mathrm{~mm} \times 20 \mathrm{~mm} \times 0.5 \mathrm{~mm}$ ), 考察其在 4 种双核功能化离子液体为催化剂的酯化体系中离子液 体的腐蚀性, 并与浓 $\mathrm{H}_{2} \mathrm{SO}_{4}$ 的催化体系进行对比 ${ }^{[19]}$. 试 样浸渍前后的处理参照国际 GB/T 4334.6-2000《不锈钢 $5 \%$ 硫酸腐蚀试验方法》进行, 实验条件为: 在 $100 \mathrm{~mL}$ 的三口烧瓶中分别加入 $0.2 \mathrm{~mol}$ 丁二酸、 $0.6 \mathrm{~mol}$ 乙醇和 一定量离子液体, $80{ }^{\circ} \mathrm{C}$ 条件下磁力搅拌 $12 \mathrm{~h}$, 得到实验 钢板反应前后的质量差. 以腐蚀率评价实验结果，腐蚀 率 $\left[\mathrm{g} /\left(\mathrm{m}^{2} \cdot \mathrm{h}\right)\right]$ 按照 Eq. 3 计算:

$$
\text { 腐蚀率 }=\frac{W_{\text {并 }}-W_{\text {后 }}}{\mathrm{S} \cdot \mathrm{t}}
$$

式中: $W$ 前为试验前试样重量, $\mathrm{g} ; W$ 后为试验后试样重量, $\mathrm{g} ; S$ 为试样总面积, $\mathrm{m}^{2} ; t$ 为试验时间, $\mathrm{h}$

\section{References}

[1] Joseph, T.; Sahoo, S.; Halligudi, S. B. J. Mol. Catal. A: Chem. 2005, 234, 107.

[2] Xie, C.; Li, H.; Li, L.; Yu, S.; Liu, F. J. Hazard. Mater. 2008, 151, 847.

[3] Li, X.; Eli, W. J. Mol. Catal. A: Chem. 2008, 279, 159.

[4] Li, X.; Lin, Q.; Ma, L. Ultrason. Sonochem. 2010, 17, 752.

[5] Sunitha, S.; Kanjilal, S.; Reddy, P. S.; Prasad, R. B. N. Tetrahedron Lett. 2007, 48, 6962.

[6] Janus, E.; Goc-Maciejewska, I.; Lozynski, M.; Pernak, J. Tetrahedron Lett. 2006, 47, 4079.

[7] Durand, J.; Teuma, E.; Gómez, M. C. R. Chim. 2007, 10, 152.

[8] Cai, S.; Wang, S. Chin. J. Chem. Eng. 2011, 19, 57. 
[9] Zhao, Y.; Li, Z.; Xia, C. Chin. J. Catal. 2011, 32, 440.

[10] Deng, Y.; Shi, F.; Beng, J.; Qiao, K. J. Mol. Catal. A: Chem. 2001, 165, 33.

[11] Fraga-Dubreuil, J.; Bourahla, K.; Rahmouni, M.; Bazureau, J. P.; Hamelin, J. Catal. Commun. 2002, 3, 185.

[12] Cole, A. C.; Jensen, J. L. J. Am. Chem. Soc. 2002, 124, 5962.

[13] Kou, Y.; Yang, Y.-L. Petrochem. Technol. 2004, 33, 297 (in Chinese).

(寇元，杨雅立，石油化工, 2004, 33, 297.)

[14] Zhang, S.-J.; Lv, X.-M. Ionic Liquid-From Basic Research to Industrial Applications, Science Press, Beijing, 2006, p. 71 (in Chinese).

(张锁江, 吕兴梅, 离子液体一从基础研究到工业应用, 科学 出版社, 北京, 2006, p. 71.)

[15] Lv, X.-M. M.S. Thesis, Nanjing University, Nanjing, 2011 (in Chi- nese).

(吕学铭, 硕士论文, 南京大学, 南京, 2011.)

[16] Bermúdez, M. D.; Jiménez, A. E.; Martínez-Nicolás, G. Appl. Surf. Sci. 2007, 253, 7295.

[17] Ganeshpure, P. A.; George, G.; Das, J. J. Mol. Catal. A: Chem. 2008, 279, 182.

[18] Liang, J.-H.; Xu, Y.; Ren, X.-Q.; Jiang, M.; Li, Z.-J. Acta PetroleI Sin. 2011, 27, 482 (in Chinese).

(梁金花，徐玥，任晓乾，姜岷，李振江，石油学报，2011，27， 482.)

[19] Huang, B.-H.; Li, Z.-J.; Shi, N.; Xu, X.-L.; Zhang, K.; Fang, Y.-X. Chin. J. Org. Chem. 2009, 29, 770 (in Chinese)

(黄宝华, 黎子进, 史娜, 徐效陵, 张焜, 方岩雄, 有机化学, 2009, 29, 770.) 